\title{
An IMPES scheme for a two-phase flow in heterogeneous porous media using a structured grid
}

\author{
Gwanghyun Jo, Do Y. Kwak ${ }^{1, *}$ \\ 291 Daehak-ro(373-1 Guseong-dong), Yuseong-gu, Daejeon 305-701, Republic of Korea
}

\begin{abstract}
We develop a numerical scheme for a two-phase immiscible flow in heterogeneous porous media using a structured grid finite element method, which have been successfully used for the computation of various physical applications involving elliptic equations [31, 30, 9, 14, 29]. The proposed method is based on the implicit pressure-explicit saturation procedure. To solve the pressure equation, we use an IFEM based on the Rannacher-Turek [35] nonconforming space, which is a modification of the work in 29] where 'broken' $P_{1}$ nonconforming element of Crouzeix-Raviart [15] was developed.

For the Darcy velocity, we apply the mixed finite volume method studied in 13, 29] on the basis of immersed finite element method (IFEM). In this way, the Darcy velocity of the flow can be computed cheaply (locally) after we solve the pressure equation. The computed Darcy velocity is used to solve the saturation equation explicitly. Thus the whole procedure can be implemented in an efficient way using a structured grid which is independent of the underlying heterogeneous porous media. Numerical results show that our method exhibits optimal order convergence rates for the pressure and velocity variables, and suboptimal rate for saturation.
\end{abstract}

Keywords: Heterogeneous porous media, Multiphase flow, Immersed finite element method, Mixed finite volume method.

\section{Introduction}

Multiphase flows in porous media arise in various disciplines including petroleum engineering, groundwater remediation, etc., see [23, 34, 4, 26, 33, 24, 38] and references therein.

Various discretization methods of the porous media problems have been developed. These methods include finite difference/volume methods 17, 38, 32, 34], and control volume methods [19, 4, 25]. Difficulties in finding accurate numerical solutions of the flows in porous media can arise if material properties change abruptly. For example, the permeability tensor often has a high variation over the space region or is even discontinuous across different materials. This makes the accurate approximation of the Darcy velocity hard, especially for the

\footnotetext{
th

*Corresponding Author. Tel: 82423502720 Fax: 82423502710 kdy@kaist.ac.kr

${ }^{1}$ This work is supported by NRF, contract No.2014R1A2A1A11053889
} 
finite difference method. Since the saturation equation is of hyperbolic type with a term including Darcy velocity, the inaccurate computation of the Darcy velocity may lead to a bad approximation of the saturation.

For an accurate approximation of Darcy velocity, mixed finite element/volume methods approach [18, 11, 10] were developed to solve the pressure equation. The mixed methods were combined with implicit pressure and explicit saturation (IMPES) scheme [39, 40] to solve the problem in an efficient way [20, 27], where the pressure and the saturation equations are solved separately.

On the other hand, discontinuous Galerkin methods which were developed for elliptic problems [41, 2] were successfully applied for these problems in 21, 22, 28]. For an excellent review for various numerical methods for solving equations involving heterogeneous porous media, see 33] and references therein.

To resolve the discontinuity across the material interface, meshes aligned with the interface are usually used for finite element method (FEM) and their variations. However, aligned meshes yield unstructured grid and as a result, the data structure becomes more complicated. Thus, the development of fast, stable numerical methods with high accuracy is an important issue for the porous media problem.

Recently, there have emerged a new method of using uniform grids for elliptic P.D.E.s with discontinuous coefficient. Immersed finite element methods (IFEM) in which one allows the interface to cut through the elements have been introduced by Z. Li, T. Lin and Y. Lin and their coworkers 31, 30], and its convergence behavior was investigated in [14]. IFEM using Crouzeix-Raviart nonconforming bases including applications to mixed finite volume method (MFVM) was developed by Kwak et al. in 29] and IFEM for nonhomogeneous jump case were considered in [9].

One of the main features of the IFEMs is that they can use any reasonable grids independent of interface. For example, some of the advantages of uniform grids for the interface problems are the following [9]:

- No grids generation is necessary which can save computation time especially for moving interface.

- It requires smaller number of degrees of freedom than fitted grids.

- Simple data structure can render easier development of fast solver such as multigrid methods or alternating direction implicit method.

- The linear systems are symmetric positive definite when the original problems are symmetric positive definite.

In this work, we introduce a numerical scheme using the IFEM for a twophase immiscible flow through heterogeneous porous media having distinct permeability. Using the concept of the global pressure of Chavent and Jaffré [10], the pressure and the saturation equations are solved separately with the IMPES scheme.

To solve the pressure equation, we adopt the MFVM introduced in [13, 29], where we integrate the momentum and mass equation directly on each element. To discretize the equation, we adopt the IFEM for Crouzeix-Raviart nonconforming element developed in 29] to the rectangular element. By choosing 
appropriate trial space (Rannacher-Turek $Q_{1}$ space) and test space (the gradient of trial space), then by eliminating the Darcy velocity from the system, we can obtain a discrete equation for pressure unknowns only.

After we solve the pressure equation, the Darcy velocity (in Raviart-Thomas [36] basis) is computed by using the local residual of the pressure equation. This approach for computing the Darcy velocity is more efficient than the classical mixed finite element method (MFEM) which yields a saddle point formulation [36], and is an alternative to the post-processing method of Arnold et al. 1]. After pressure equation is solved, saturation equation is solved by the control volume method [37, 12] together with the upwind scheme. Numerical examples show that our scheme gives optimal order convergence for both pressure and velocity for all examples we tested. For saturation, we obtain the errors at least $O\left(h^{1.5}\right)$ in $L^{2}$-norm and $O(h)$ in $H^{1}$-norm for the case of zero capillary and around $O\left(h^{1.5}\right)$ in $L^{2}$-norm and $O\left(h^{0.5}\right)$ in $H^{1}$-norm for the nonzero capillary case.

The rest of the paper is organized as follows. The governing equations of two phase immiscible flows in heterogeneous porous media are described in section 2. In section 3, we present a brief review of IFEM and MFVM for second order elliptic equations. In section 4, we describe our version of IMPES for two phase flows in heterogeneous porous media. Numerical examples are given in section 5 , and conclusion follows in section 6 .

\section{Model problem}

Let $\Omega=\Omega^{+} \cup \Omega^{-}$be a polygonal domain in $\mathbb{R}^{2}$ separated by a $C^{1}$-interface $\Gamma$ (See figure 1). We assume the subdomains $\Omega^{+}$and $\Omega^{-}$are occupied by heterogeneous media having discontinuous permeability tensor $\mathbf{K}$. The flows of wetting phase and non-wetting phases, i.e. $\alpha \in\{n, w\}$ in $\Omega$ are described by Darcy's law and the continuity equation for each phase. Governing equations for two-phase flow in porous media, in the absence of gravity, for time $[0, T]$ is given by

$$
\frac{\partial\left(\Phi \rho_{\alpha} S_{\alpha}\right)}{\partial t}+\nabla \cdot\left\{\rho_{\alpha} \mathbf{u}_{\alpha}\right\}-\rho_{\alpha} q_{\alpha}=0, \quad \alpha=w, n,
$$

where $\mathbf{u}_{\alpha}$ are the Darcy velocities given by

$$
\mathbf{u}_{\alpha}=-\lambda_{\alpha} \mathbf{K} \nabla p_{\alpha},
$$

saturations $S_{\alpha}$ and pressures $p_{\alpha}$ satisfy the relations:

$$
\begin{aligned}
S_{w}+S_{n} & =1 \\
p_{n}-p_{w} & =p_{c}\left(S_{w}\right) .
\end{aligned}
$$

Here, $\lambda_{\alpha}$ is mobility, $\rho_{\alpha}$ is density and $q_{\alpha}$ is source term for the phase $\alpha \in\{w, n\}$. Mobility $\lambda_{\alpha}$ is defined by $\lambda_{\alpha}=k_{r \alpha} / \mu_{\alpha}$, where $k_{r \alpha}$ is relative permeability and $\mu_{\alpha}$ is the viscosity. $p_{c}$ is the capillary pressure, $\Phi$ is the porosity and $\mathbf{K}$ is the permeability tensor. The permeability $\mathbf{K}$ is symmetric positive definite and mobilities $\lambda_{\alpha}$ is nonnegative for both phase $\alpha \in\{w, n\}$. We consider the incompressible fluid where $\Phi$ and $\rho_{\alpha}$ is constant and we neglect the effect of gravity. 


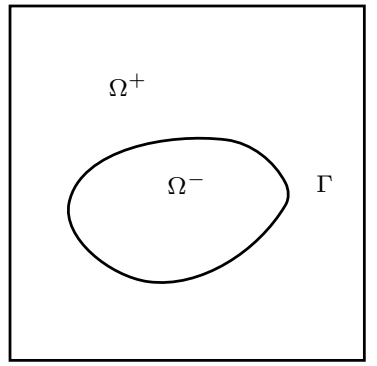

Figure 1: A domain $\Omega$ with interface

To solve (11), (2) we adopt the concept of reformulation by Chavent and Jaffré [10] where new primary variable global pressure is introduced. Total velocity $\mathbf{u}$ is defined by sum of $\mathbf{u}_{w}$ and $\mathbf{u}_{n}$

$$
\mathbf{u}=\mathbf{u}_{n}+\mathbf{u}_{w}=-\lambda \mathbf{K}\left(\nabla p_{n}-f_{w} \nabla p_{c}\right)
$$

where total mobility $\lambda$, fractional flow $f_{\alpha}$ are defined by

$$
\lambda=\lambda_{w}+\lambda_{n}, \quad f_{\alpha}=\frac{\lambda_{\alpha}}{\lambda} .
$$

We assume that $\lambda$ is strictly positive. In [10], global pressure is defined in such a way that:

$$
\nabla p=\nabla p_{n}-f_{w} \nabla p_{c}
$$

leading to the relation of total velocity and global pressure:

$$
\mathbf{u}=-\lambda K \nabla p
$$

The model problem can be written in an alternative way with unknowns $p$ and $S_{w}$

$$
\begin{aligned}
-\nabla \cdot(\lambda \mathbf{K} \nabla p) & =q_{w}+q_{n}, \\
\Phi \frac{S_{w}}{\partial t} & =q_{w}-\nabla \cdot\left(f_{w} \mathbf{u}+\lambda_{n} f_{w} \mathbf{K} \nabla p_{c}\right) .
\end{aligned}
$$

Initial conditions and boundary conditions are given as follows:

$$
\begin{array}{lll}
S_{w}(\mathbf{x}, 0)=S_{w 0}(\mathbf{x}), & \mathbf{x} \in \Omega & \\
p(\mathbf{x}, t)=p_{d}(\mathbf{x}, t) \quad \text { on } & \Gamma_{\mathrm{d}} & \mathbf{u} \cdot \mathbf{n}=U(\mathbf{x}, t) \text { on } \Gamma_{\mathrm{n}} \\
S_{w}(\mathbf{x}, t)=S_{w d}(\mathbf{x}, t) \quad \text { on } \quad \Gamma_{\mathrm{wd}} & \mathbf{u}_{w} \cdot \mathbf{n}=U_{w}(\mathbf{x}, t) \quad \text { on } \Gamma_{\mathrm{wn}}
\end{array}
$$
(4).

The goal of this work is to provide numerical methods for the problem (3),

\section{MFVM and IFEM for second order elliptic equations}

In this section, we briefly explain MFVM [13] and IFEM [29] for elliptic problems having discontinuous coefficients. The IFEM was originally designed 
for $P_{1}$ nonconforming element of Crouzeix-Raviart, but we modify it to the case of $Q_{1}$-nonconforming element of Rannacher-Turek [35].

Consider the second order elliptic model problem,

$$
\left\{\begin{array}{rlll}
-\operatorname{div}(\beta \nabla \mathrm{p}) & =f & \text { in } & \Omega \\
p & =0 & \text { on } & \partial \Omega
\end{array}\right.
$$

where $f \in L^{2}(\Omega)$ and $p \in H_{0}^{1}(\Omega)$ and $\beta$ is symmetric and uniformly positive definite matrix with possibly discontinuous entries on the domain $\Omega$.

\section{1. $M F V M$}

In the computation of porous media equation, it is important to evaluate Darcy velocity accurately, which is a reason for using the MFEM [5, 6, 1, 36, 7, 16]. Raviart-Thomas element [36] and Brezzi-Douglas-Marini element [6] are most widely used ones among many MFEMs. However, one disadvantage of the MFEM formulation is that it leads to a saddle point problem. One way to avoid the saddle point formulation is the post processing technique of Arnold and Brezzi [1]. An alternative, in view of finite volume methods, was suggested by Chou, Kwak and Kim [13] where the equations in the mixed form are integrated against the gradient of test functions on each element. We explain it here. By introducing the velocity $\mathbf{u}=-\beta \nabla p$, we rewrite (5) in a mixed form

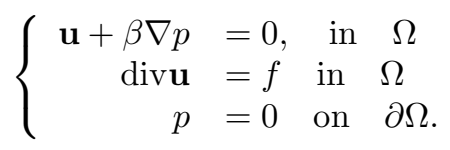

For simplicity, assume $\Omega$ is a rectangle and let $\mathcal{T}_{h}$ be a triangulation by partitioned by rectangles, the case of triangles can be similarly treated.

For the basis functions, we use the rotated- $Q_{1}$ nonconforming finite elements [35] for the pressure and the lowest-order Raviart-Thomas elements [36] for the velocity. The local space for pressure on the reference element is given by

$$
N_{h}(\hat{Q})=\operatorname{span}\left\{1, \hat{x}, \hat{y}, \hat{x}^{2}-\hat{y}^{2}\right\}
$$

where the degrees of freedoms are the average values on the edges, i.e., $\left\{\frac{1}{|\hat{e}|} \int_{\hat{e}} \hat{p}_{h} d s\right.$ : $\hat{e}$ is and edge of $\left.\mathcal{T}_{h}\right\}$. The local space for velocity is defined by

$$
V_{h}(\hat{Q})=\{\hat{v}: \hat{v}=(a+b \hat{x}, c+d \hat{y}), a, b, c, d \in \mathbb{R}\}
$$

and the actually elements are defined through the Piola mapping. The global spaces $V_{h}(\Omega)$ and $N_{h}(\Omega)$ are defined in an obvious way.

The MFVM scheme for (6) is the following: Find $\left(\mathbf{u}_{h}, p_{h}\right) \in V_{h}(\Omega) \times N_{h}(\Omega)$, which satisfies

$$
\left\{\begin{aligned}
\int_{Q}\left(\mathbf{u}_{h}+\beta \nabla p_{h}\right) \cdot \nabla \chi & =0, \quad \forall \chi \in N_{h}(Q), \\
\int_{Q} \operatorname{div} \mathbf{u}_{\mathrm{h}} & =\int_{Q} f
\end{aligned}\right.
$$


for every element $Q \in \mathcal{T}_{h}$. This system has an unique solution $\left(\mathbf{u}_{h}, p_{h}\right) \in$ $V_{h}(\Omega) \times N_{h}(\Omega)$ and the following optimal error estimates holds [13]

$$
\begin{aligned}
& \left\|p-p_{h}\right\|_{L^{2}(\Omega)}+h\left|p-p_{h}\right|_{H^{1}(\Omega)} \leq C h^{2}\|f\|_{H^{1}(\Omega)} \\
& \left\|\mathbf{u}-\mathbf{u}_{h}\right\|_{L^{2}(\Omega)}+\left\|\operatorname{div} \mathbf{u}-\operatorname{div} \mathbf{u}_{h}\right\|_{L^{2}(\Omega)} \leq C h\left(\|\mathbf{u}\|_{H^{1}(\Omega)}+\|f\|_{H^{1}(\Omega)}\right) .
\end{aligned}
$$

Even though the equation (7) is hard to implement, a nice thing about it is that we can transform it to the primal form for $p_{h}$ only. Since $\mathbf{u}_{h} \cdot \mathbf{n}$ is constant on the edges and $\chi \in N_{h}(\Omega)$ has common averages on the interior edges, we obtain for each $\chi \in N_{h}(\Omega)$,

$$
\sum_{Q \in \mathcal{T}_{h}} \int_{Q} \mathbf{u}_{h} \cdot \nabla \chi=\sum_{Q \in \mathcal{T}_{h}}\left[\int_{\partial Q}\left(\mathbf{u}_{h} \cdot n\right) \chi-\int_{Q} \operatorname{div} \mathbf{u}_{\mathrm{h}} \chi\right]=-\int_{Q}\left(\left.\bar{f}_{h}\right|_{Q}\right) \chi,
$$

where $\left.\bar{f}_{h}\right|_{Q}$ is the local average of $f$ on $Q$. Considering (7) we obtain

$$
\sum_{Q \in \mathcal{T}_{h}} \int_{Q} \beta \nabla p_{h} \cdot \nabla \chi=\int_{\Omega} \bar{f}_{h} \chi, \quad \chi \in N_{h}
$$

which is a symmetric, positive definite system that can be solved easily by conjugate gradient (CG) or preconditioned conjugate gradient (PCG) methods.

Once $p_{h}$ is obtained, the velocity $\mathbf{u}_{h}$ can be computed locally. Let $Q \in \mathcal{T}_{h}$ has edges $e_{i}, i=1,2,3,4$ and $\phi_{i} \in N_{h}(Q)$ be the basis function associated with the edge $e_{i}$. Then the normal components of velocity are computed locally as follows [13]:

$$
\left.\left|e_{i}\right|\left(\mathbf{u}_{h} \cdot \mathbf{n}\right)\right|_{e_{i}}=\int_{Q} \bar{f}_{h} \phi_{i}-\int_{Q} \beta \nabla p_{h} \cdot \nabla \phi_{i} .
$$

We introduce function spaces, norms, etc, necessary for error analysis. For any domain $D$, and $m=0,1, \cdots$, we let $W_{p}^{m}(D)$ be the usual Sobolev space with norms(semi-norms) denoted by $\|\cdot\|_{m, p, D}\left(|\cdot|_{m, p, D}\right)$. When $p=2$, we write $H^{m}(D):=W_{2}^{m}(D)$ with the norms (semi-norms) $\|\cdot\|_{m, D}\left(|\cdot|_{m, D}\right)$. Let $H_{0}^{1}(\Omega)$ be the subspace of $H^{1}(\Omega)$ with zero trace on the boundary. We also define, for $m=1,2$

$$
\widetilde{H}^{m}(D):=\left\{u \in H^{m-1}(D):\left.u\right|_{D \cap \Omega^{s}} \in H^{m}\left(D \cap \Omega^{s}\right), s=+,-\right\}
$$

with norms,

$$
\begin{aligned}
|u|_{m, D}^{2} & :=|u|_{m, D \cap \Omega^{+}}^{2}+|u|_{m, D \cap \Omega^{-}}^{2} \\
\|u\|_{m, D}^{2} & :=\|u\|_{m, D \cap \Omega^{+}}^{2}+\|u\|_{m, D \cap \Omega^{-}}^{2}
\end{aligned}
$$

We let $H_{h}(\Omega):=H_{0}^{1}(\Omega)+N_{h}(\Omega)$ equipped with norms $|u|_{1, h}:=\left(\sum_{Q}|u|_{1, Q}^{2}\right)^{1 / 2}$ and $\|u\|_{1, h}:=\left(\sum_{Q}\|u\|_{1, Q}^{2}\right)^{1 / 2}$.

\subsection{IFEM for problems with discontinuous coefficients}

In this subsection, we briefly review the IFEM for problem with discontinuous coefficients. This method allows us to use the grid that does not align with the interface. We restrict our attention to the case of scalar $\beta$. Tensor coefficient can be treated similarly. 
Assume

$$
\beta= \begin{cases}\beta^{+}, & \mathbf{x} \in \Omega^{+} \\ \beta^{-}, & \mathbf{x} \in \Omega^{-}\end{cases}
$$

with jump conditions

$$
[p]=0,\left[\beta \frac{\partial p}{\partial n}\right]=0 \quad \text { across } \Gamma .
$$

The IFEMs have been suggested and proven to be efficient for elliptic problems, see the work of Z. Li, T. Lin and Y. Lin, etc., in [31, 30] and S. Chou, D. Y. Kwak and K. T. Wee in [14, 29]. In particular, the edge based Crouzeix-Raviart $P_{1}$-nonconforming IFEM combined with Raviart-Thomas MFVM [29] turns out to be efficient in implementing mixed formulation through a primary form.

We develop the $Q_{1}$-nonconforming version of IFEM scheme. To do so, we modify Rannacher-Turek element [35] $Q_{1}$-nonconforming basis function so that the flux is weakly continuous along the local interface in a manner similar to [29]. To do so, we begin need to explain the interface/element relation: We call

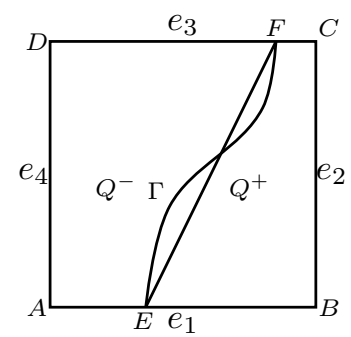

Figure 2: A typical interface rectangular

an element $Q \in \mathcal{T}_{h}$ is an interface element if the interface $\Gamma$ passes though the interior of $Q$ (see Figure 2). Suppose interface cut through the edges of $Q$ at point $E$ and $F$ and divide $Q$ by two section $Q^{+}$and $Q^{-}$. Let $G$ be the midpoint of $\overline{E F}$ Let $e_{i}, i=1,2,3,4$, be the edges of $Q$. For $\phi \in H^{1}(T)$, let $\bar{\phi}_{e_{i}}$ denote the average of $\phi$ along $e_{i}$, i.e.,

$$
\bar{\phi}_{e_{i}}:=\frac{1}{\left|e_{i}\right|} \int_{e_{i}} \phi d s .
$$

We construct a basis function of the form

$$
\phi= \begin{cases}\phi^{+}=a_{0}+b_{0} x+c_{0} y+d_{0}\left(x^{2}-y^{2}\right), & (x, y) \in Q^{+} \\ \phi^{-}=a_{1}+b_{1} x+c_{1} y+d_{1}\left(x^{2}-y^{2}\right), & (x, y) \in Q^{-}\end{cases}
$$

satisfying

$$
\begin{aligned}
\bar{\phi}_{e_{i}} & =V_{i}, \quad i=1,2,3,4, \\
\phi^{+}(E) & =\phi^{-}(E), \quad \phi^{+}(F)=\phi^{-}(F), \\
d_{0} & =d_{1}, \\
\beta^{+} \frac{\partial \phi^{+}}{\partial n_{\overline{E F}}}(G) & =\beta^{-} \frac{\partial \phi^{-}}{\partial n_{\overline{E F}}}(G),
\end{aligned}
$$


where $V_{i}, i=1,2,3,4$ are given values, $n \overline{E F}$ is the unit normal vector on the line segment $\overline{E F}$. It is easy to see the conditions (11), (12), (13) and (14) determine $\phi$ uniquely. Let $\widehat{N}_{h}(Q)$ denote the (local) four-dimensional space spanned by these shape functions.

We define the immersed finite element space $\widehat{N}_{h}(\Omega)$ as the collection of the functions such that

$$
\begin{cases}\left.\phi\right|_{Q} \in N_{h}(Q) & \text { if } Q \text { is a noninterface element, } \\ \left.\phi\right|_{Q} \in \widehat{N}_{h}(Q) & \text { if } Q \text { is an interface element, } \\ \left.\int_{e} \phi\right|_{Q_{1}} d s=\left.\int_{e} \phi\right|_{Q_{2}} d s & \text { if } Q_{1}, Q_{2} \text { are adjacent elements and } e \text { is a common edge, } \\ \int_{e} \phi d s=0 & \text { if } e \text { is part of the boundary } \partial \Omega\end{cases}
$$

The IFEM scheme for the equation (5) is given as follows: Find $p_{h} \in \widehat{N}_{h}(\Omega)$ such that

$$
a_{h}\left(p_{h}, \phi\right)=(f, \phi), \quad \forall \phi \in \widehat{N}_{h}(\Omega)
$$

where

$$
\begin{aligned}
a_{h}(q, \phi) & =\sum_{Q \in \mathcal{T}_{h}} \int_{Q} \beta \nabla q \cdot \nabla \phi d x, \quad \forall q, \phi \in \widehat{H}_{h}(\Omega), \\
\widehat{H}_{h}(\Omega) & :=H_{0}^{1}(\Omega)+\widehat{N}_{h}(\Omega) .
\end{aligned}
$$

Here $\widehat{H}_{h}(\Omega)$ is equipped with the norm $\|\cdot\|_{1, h}$. A slight modification of the proof in [29] gives the following result.

Proposition 3.1. Suppose $p \in \widetilde{H}^{2}(\Omega)$ and $p_{h} \in \widehat{N}_{h}(\Omega)$ are solutions to (6) and (15). Then the following error estimates holds:

$$
\left\|p-p_{h}\right\|_{L^{2}(\Omega)}+h\left|p-p_{h}\right|_{1, h} \leq C h^{2}\|p\|_{\widetilde{H}^{2}(\Omega)} .
$$

\subsection{MFVM using IFEM}

We apply our IFEM to MFVM introduced in Section 3.1 to compute Darcy velocity. The method is similar to MFVM introduced in section 3.1., but the $Q_{1}$-nonconforming finite element space $N_{h}(\Omega)$ is replaced by broken $Q_{1^{-}}$ nonconforming space $\widehat{N}_{h}(\Omega)$ in (7) and we obtain the following error estimate:

\section{Proposition 3.2.}

$$
\begin{aligned}
& \left\|p-p_{h}\right\|_{L^{2}(\Omega)}+h\left|p-p_{h}\right|_{1, h} \leq C h^{2}\|p\|_{\widetilde{H}^{2}(\Omega)} \\
& \left\|\mathbf{u}-\mathbf{u}_{h}\right\|_{L^{2}(\Omega)}+\left\|\operatorname{div} \mathbf{u}-\operatorname{div} \mathbf{u}_{h}\right\|_{L^{2}(\Omega)} \leq C h\left\{\|\mathbf{u}\|_{H^{1}(\Omega)}+\|p\|_{\widetilde{H}^{2}(\Omega)}+\|f\|_{\widetilde{H}^{1}(\Omega)}\right\}
\end{aligned}
$$

provided $\mathbf{u} \in \mathbf{H}^{1}(\Omega)$ and $f \in \widetilde{H}^{1}(\Omega)$.

\section{Application to IMPES scheme}

Suppose the permeability tensor given in the form $\mathbf{K}=K \mathbf{I}$, where

$$
K= \begin{cases}K^{+}, & \mathbf{x} \text { in } \Omega^{+} \\ K^{-}, & \mathbf{x} \text { in } \Omega^{-}\end{cases}
$$


We will briefly review the original IMPES introduced by Sheldon (1959) and Stonde and Gardner (1961) 39, 40]. Consider the problem (3), (44) on the time interval $[0, T]$. Let $\triangle t>0$ be a timestep and divide $T$ by $N$ time steps. Denote $t^{l}=l \Delta t$ for $l=0,1,2, \ldots, N$. Equations (3)-(41) are solved sequentially: Given the saturation at time level $t^{l}$, the pressure $p$ at time $t^{l+1}$ is computed from (3) which is a linear equation in $p$. Then the saturation at time level $t^{l+1}$ is computed from (4) with velocity fields $\mathbf{u}$ obtained at time level $t^{l+1}$.

\section{IMPES Algorithm.}

For $l=0, \cdots, N-1$, repeat the following process.

1. Solve for $p^{l+1}$ and $\mathbf{u}^{l+1}$ with given $S_{w}^{l}$

$$
\left\{\begin{aligned}
\nabla \cdot \mathbf{u}^{l+1} & =q_{w}^{l+1}+q_{n}^{l+1} \\
\mathbf{u}^{l+1} & =-\lambda^{l} \mathbf{K} \nabla p^{l+1}
\end{aligned}\right.
$$

where $\lambda^{l}=\lambda\left(S_{w}^{l}\right)$.

2. Compute $S_{w}^{l+1}$

$$
\left\{\begin{aligned}
\mathbf{u}_{w}^{l+1} & =f_{w}^{l} \mathbf{u}^{l+1}+\lambda_{n}^{l} f_{w}^{l} \mathbf{K} \nabla p_{c}\left(S_{w}^{l}\right) \\
\Phi\left(S_{w}^{l+1}-S_{w}^{l}\right) & =\Delta t\left(q_{w}^{l+1}-\nabla \cdot \mathbf{u}_{w}^{l+1}\right)
\end{aligned}\right.
$$

where $f_{w}^{l}=f_{w}\left(S_{w}^{l}\right)$.

\section{3. $l=l+1$}

We will call (19) the pressure equation, and (20) the saturation equation. In our version of IMPES, we solve the pressure equation by MFVM based on IFEM introduced in Section 3.3 and then solve the saturation equation by the control volume method.

\subsection{Pressure equation}

Since we use saturation $S_{w}$ at time level $t^{l}, \lambda^{l}$ is considered as a coefficient of the pressure equation.

Since global pressure and total velocity are continuous along the interface $\Gamma$, we impose the condition

$$
\left[p^{l+1}\right]=0,\left[K \frac{\partial p^{l+1}}{\partial n}\right]=0 \quad \text { across } \quad \Gamma .
$$

This can be effectively solved by the IFEM described in section 3.2: Find $p_{h}^{l+1} \in$ $\widehat{N}_{h}(\Omega)$ satisfying

$$
a_{h}\left(p_{h}^{l+1}, \phi\right)=\left(q_{w}^{l+1}+q_{n}^{l+1}, \phi\right), \quad \forall \phi \in \widehat{N}_{h}(\Omega)
$$

where is the bilinear form $a_{h}(\cdot, \cdot)$ is same as (16) except that the coefficient $\beta$ is replaced by $\lambda^{l} \mathbf{K}$.

Once the global pressure $p_{h}^{l+1}$ is solved by IFEM, the total velocity $\mathbf{u}_{h}^{l+1}$ is computed by local recovery technique of MFVM in Section 3.3. 


\subsection{Saturation equation}

First we note that the $Q_{1}$-nonconforming element can have negative values even though the degrees of freedom are nonnegative. Since the saturation variables $S_{\alpha}$ cannot have negative values, it is inappropriate to use the the $Q_{1}$ nonconforming element. Hence we use the $Q_{1}$-conforming finite element for the approximation of the saturation.

Let $U_{h}(\Omega)$ denote $Q_{1}$-conforming finite element space defined over the triangulation given above. We apply the control volume method to (20) together with the upwinding scheme as used in [19, 4, 25], which we briefly explain as follows: Fix an interior vertex $P$ and consider the four elements $Q_{1}, Q_{2}, Q_{3}$ and $Q_{4}$ sharing $P$ as a common vertex. Connecting the centers of the four elements $Q_{i}, i=1, \cdots, 4$, we obtain a dual volume, which we shall call $Q_{P}^{*}$ (Figure 3). For a boundary vertex, an obvious modification is necessary.

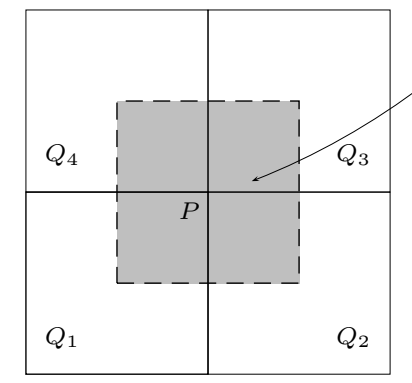

$Q_{P}^{*}$

Figure 3: A typical dual volume $Q_{P}^{*}$ associated with node $P$.

The collection of dual volumes $Q_{P}^{*}$ is denoted by $\mathcal{D}_{h}$.

A test function space $W_{h}(\Omega)$ using $D_{h}$ by

$$
W_{h}(\Omega)=\left\{\psi \in L^{2}(\Omega) \mid \psi \text { is piecewise constant on each } Q_{P}^{*} \in \mathcal{D}_{h}\right\} .
$$

To compute $S_{w}^{l+1}$ in (20), we first consider the Petrov-Galerkin methods with test function space $W_{h}(\Omega)$ : Find $S_{w}^{l+1} \in U_{h}$ such that satisfies

$$
\int_{\Omega} \Phi S_{w}^{l+1} \psi_{i}=\int_{\Omega}\left(\Phi S_{w}^{l}+\triangle t q_{w}^{l+1}\right) \psi_{i}-\int_{\Omega} \Delta t \nabla \cdot \mathbf{u}_{w}^{l+1} \psi_{i}
$$

for all $\psi_{i} \in W_{h}(\Omega)$. Fix a vertex $P_{i}$ shared by four neighboring elements $Q_{k}, k=1, \cdots, 4$ and applying the divergence theorem in (22) on $Q_{P_{i}}^{*}$ leads to the following form

$$
\int_{Q_{P_{i}}^{*}} S_{w}^{l+1}=\int_{Q_{P_{i}}^{*}}\left(S_{w}^{l}+\frac{\triangle t}{\Phi} q_{w}^{l+1}\right)-\frac{\triangle t}{\Phi} \int_{\partial Q_{P_{i}}^{*}} \mathbf{u}_{w}^{l+1} \cdot \mathbf{n} d s
$$

Now we approximate the second term of (23) by the upwinding concept. Suppose $P_{j}$ is an adjacent node of $P_{i}$ belonging to the element $Q_{k}$ with barycenter $C_{k}$. We denote by $M_{i j}$ the mid point of $P_{i}$ and $P_{j}$. Let us denote by $\gamma_{i j}^{k}$ the segment $\overline{M_{i j} C_{k}}$, whose outward normal vector is $\mathbf{n}_{i j}^{k}$. Then,

$$
\int_{\partial Q_{P_{i}}^{*}} \mathbf{u}_{w}^{l+1} \cdot \mathbf{n} d s=\sum_{k=1}^{4} \int_{\partial Q_{P_{i}}^{*} \cap Q_{k}} \mathbf{u}_{w}^{l+1} \cdot \mathbf{n} d s=\sum_{k=1}^{4} \sum_{j=j_{1}^{k}, j_{2}^{k}} \int_{\gamma_{i j}^{k}} \mathbf{u}_{w}^{l+1} \cdot \mathbf{n}_{i j}^{k} d s,
$$


where $P_{j_{1}^{k}}$ and $P_{j_{2}^{k}}$ are two adjacent nodes of $P_{i}$, belonging to the element $Q_{k}$ (see Figure 4). Noting that

$$
\int_{\gamma_{i j}^{k}} \mathbf{u}_{w}^{l+1} \cdot \mathbf{n}_{i j}^{k} d s=\int_{\gamma_{i j}^{k}} f_{w}\left(S_{w}\right)\left(\mathbf{u}^{l+1}+K \lambda_{n}^{l} \nabla p_{c}\left(S_{w}^{l}\right)\right) \cdot \mathbf{n}_{i j}^{k} d s,
$$

we replace it by

$$
\int_{\gamma_{i j}^{k}} f_{w}\left(S_{w}^{*}\right)\left(\mathbf{u}^{l+1}+K \lambda_{n}^{l} \nabla p_{c}\left(S_{w}\right)^{l}\right) \cdot \mathbf{n}_{i j}^{k} d s
$$

where

$$
S_{w}^{*}=\left\{\begin{array}{lll}
S_{w}\left(P_{i}\right), & \text { if } \quad\left(\mathbf{u}^{l+1}+K \lambda_{n}^{l} \nabla p_{c}\left(S_{w}\right)^{l}\right) \cdot \mathbf{n}_{i j}^{k} \geq 0 \\
S_{w}\left(P_{j}\right), & \text { if } \quad\left(\mathbf{u}^{l+1}+K \lambda_{n}^{l} \nabla p_{c}\left(S_{w}\right)^{l}\right) \cdot \mathbf{n}_{i j}^{k}<0
\end{array}\right.
$$

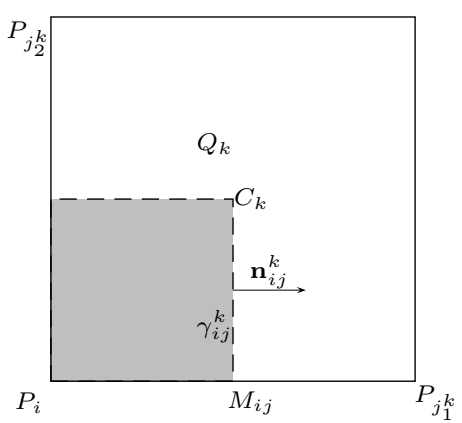

Figure 4: Illustration of $\gamma_{i j}^{k}$ in $Q_{k}$ for $j=j_{1}^{k}$.

The final scheme for the saturation equation is as follows: Find $S_{w}^{l+1} \in U_{h}(\Omega)$ such that:

$$
\int_{Q_{P_{i}}^{*}} S_{w}^{l+1}=\int_{Q_{P_{i}}^{*}}\left(S_{w}^{l}+\frac{\triangle t}{\Phi} q_{w}^{l+1}\right)-\frac{\triangle t}{\Phi} \sum_{k} \sum_{j=j_{1}^{k}, j_{2}^{k}} \int_{\gamma_{i j}^{k}} f_{w}\left(S_{w}^{*}\right)\left(\mathbf{u}^{l+1}+K \lambda_{n}^{l} \nabla p_{c}\left(S_{w}\right)^{l}\right) \cdot \mathbf{n}_{i j}^{k} d s,
$$

for all $P_{i}$.

\section{Numerical Results}

We present some numerical simulations of two phase immiscible flows in porous media. For the first three examples, the exact solutions are known and the domain $\Omega$ is $[0, \pi / 2]^{2}$. The fourth example is the famous five spot flooding problem with $\Omega=(0,300[\mathrm{~m}]) \times(0,300[\mathrm{~m}])$.

We divide the domain by $n \times n\left(n=2^{j}(j=2,3, \cdots)\right)$ rectangles whose sides are parallel to the coordinate axes. The relative permeability $k_{r \alpha}$ is given by Brooks-Corey model with index $\lambda=2[8]$ :

$$
\left\{\begin{array}{l}
k_{r w}\left(S_{w}\right)=S_{w}^{4} \\
k_{r n}\left(S_{w}\right)=\left(1-S_{w}\right)^{2}\left(1-S_{w}^{2}\right) .
\end{array}\right.
$$


In first two examples, we assume zero capillary effects. In example 5.3, we consider the case of capillary pressure given by Brooks-Corey model with index $\lambda=2$ :

$$
p_{c}\left(S_{w}\right)=p_{d} S_{w}^{-\frac{1}{2}},
$$

where $p_{d}$ is an entry pressure.

We use the level set function

$$
L(x, y): \Omega \rightarrow \mathbb{R}
$$

to describe the interface between the heterogeneities of the domain. We assume that

$$
L(x, y)=\left\{\begin{array}{rrrr}
<0, & (x, y) & \text { in } & \Omega^{-} \\
0, & (x, y) & \text { on } & \Gamma \\
>0, & (x, y) & \text { in } & \Omega^{+}
\end{array}\right.
$$

and the permeability $K$ is given as follows:

$$
K(x, y)=\left\{\begin{array}{llll}
K^{+}, & (x, y) & \text { in } & \Omega^{+} \\
K^{-}, & (x, y) & \text { in } & \Omega^{-} .
\end{array}\right.
$$

Our examples are constructed so that fluxes are continuous along the interface $\Gamma$, i.e. $[\mathbf{u} \cdot \mathbf{n}]=\left[\mathbf{u}_{w} \cdot \mathbf{n}\right]=0$ across $\Gamma$.

Example 5.1. We set the level set function $L(x, y)$ as

$$
L(x, y)=(x+y-1)(x+y-3) .
$$

Permeability is given by $K^{+}=1$ and $K^{-}=0.001$. The exact solutions are given as follows:

$$
\begin{aligned}
& p(x, y, t) \\
& = \begin{cases}\left(l_{x y}-1\right)\left(l_{x y}-2\right)(2-t) K^{-}+100, & 0 \leq l_{x y}<1 \\
-\left(l_{x y}-1\right)\left\{1 / 3\left(l_{x y}-1\right)^{2}-\left(l_{x y}-1\right)+1\right\}(2-t) K^{+}+100, & 1 \leq l_{x y} \leq 3, \\
-\left\{\left(l_{x y}-3\right)\left(l_{x y}-2\right)+\frac{2}{3}\right\}(2-t) K^{-}+100, & 3 \leq l_{x y} \leq \pi,\end{cases}
\end{aligned}
$$

and

$$
S_{w}(x, y, t)=\left(1-1 / 8 l_{x y}-1 / 20 l_{x y}^{2}\right)(0.5+0.5 t),
$$

where $l_{x y}=x+y$ and the source terms $q_{w}$ and $q_{n}$ are chosen to satisfy (3) and (4).

We set the porosity $\Phi=1$ and the viscosities $\mu_{w}=\mu_{n}=1$. Dirichlet boundary condition on all of $\partial \Omega$ is imposed.

Table 1 shows the $L^{2}, H^{1}$ errors of saturation, pressure and Darcy velocity when $T=1$. The time step $\triangle t$ is chosen as $\triangle t=16 / n^{2}=\left(64 / \pi^{2}\right) h^{2}$.

Example 5.2. We set the level set function $L(x, y)$ as

$$
L(x, y)=(x-0.5)^{2}+(y-0.5)^{2}-\frac{1}{16} .
$$


Table 1: MFVM based on broken $Q_{1}$-nonconforming IFEM methods for Example 5.1

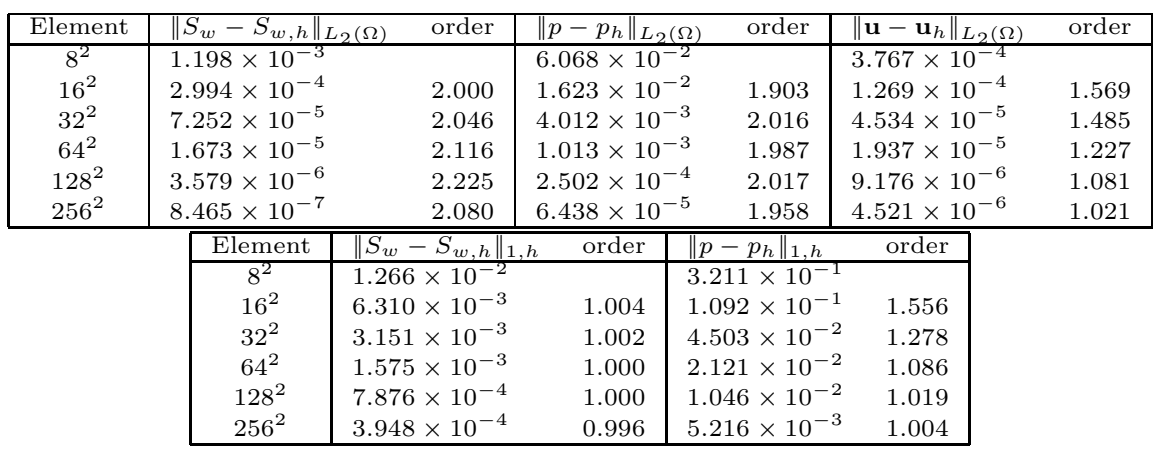

Table 2: MFVM based on broken $Q_{1}$-nonconforming IFEM methods for Example 5.2

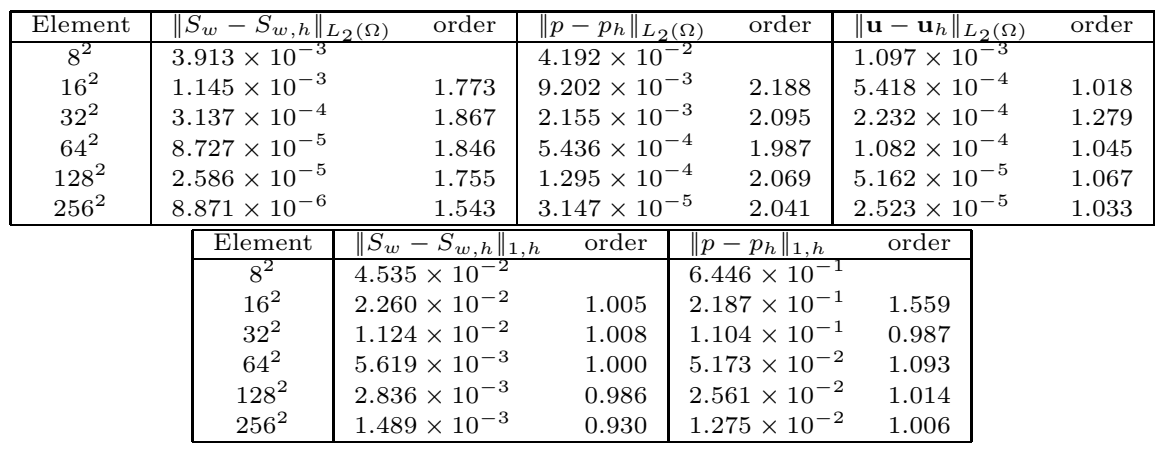

Permeability is $K^{+}=1$ and $K^{-}=0.001$. The exact solutions are given as:

$$
\begin{aligned}
p(x, y, t) & = \begin{cases}-10 L(x, y)(x-1)(2-t) K^{-}+100, & L(x, y)>0 \\
10\left(1-e^{L(x, y)(x-1)}\right)(2-t) K^{+}+100, & L(x, y) \leq 0,\end{cases} \\
S_{w}(x, y, t) & =\cos x(0.2+0.5 t),
\end{aligned}
$$

where the source terms $q_{\alpha}$, porosity and viscosities are the same as Example 5.1. Dirichlet boundary condition on all of $\partial \Omega$ is imposed.

Table 2 shows the $L^{2}, H^{1}$ errors of saturation, pressure and Darcy velocity when $T=1$. The time step $\triangle t$ is chosen as $\triangle t=16 / n^{2}=\left(64 / \pi^{2}\right) h^{2}$.

Example 5.1 and Example 5.2 show second order convergence in $L^{2}$-norm and first order in $H^{1}$-norm for the pressure variable, and first order in $L^{2}$-norm for the velocity variable. At least one and half order is observed in $L^{2}$-norm and first order in $H^{1}$-norm for the saturation.

Example 5.3. We set the level set function $L(x, y)$ as

$$
L(x, y)=(2-x-y)
$$


Table 3: MFVM based on broken $Q_{1}$-nonconforming IFEM methods for Example 5.3 with permeability $\left(K^{+}=0.02, K^{-}=0.008\right)$

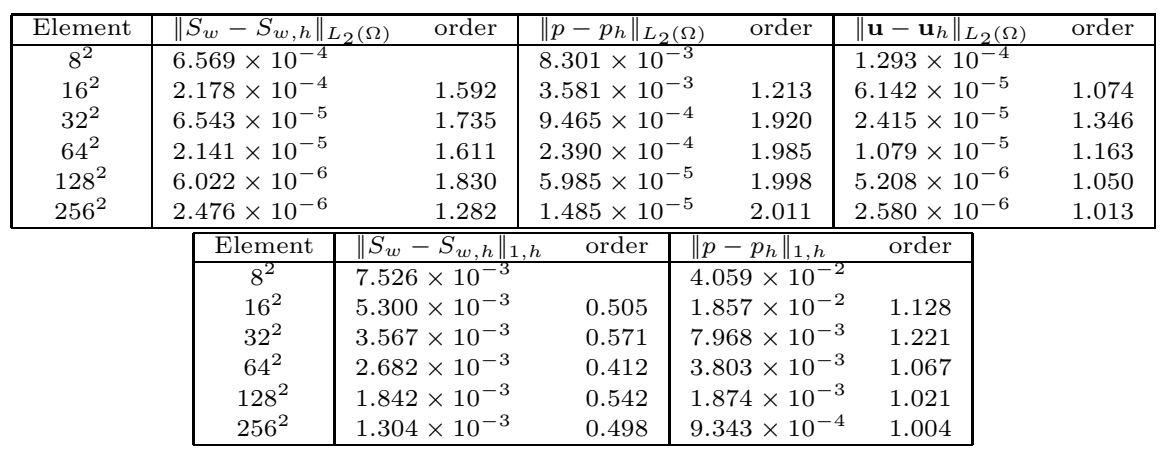

The exact solutions are given as:

$$
\begin{aligned}
p(x, y, t) & = \begin{cases}-10 L \cos \left(l_{x y}\right)(2-t) K^{-}+100, \quad L(x, y)>0 \\
-10 L \cos \left(l_{x y}\right)(2-t) K^{+}+100, \quad L(x, y) \leq 0,\end{cases} \\
S_{w}(x, y, t) & =\left\{\begin{array}{ll}
\left(1-4\left(l_{x y}+0.25\left(l_{x y}-2\right)^{2}\right) K^{-}\right)(1-0.5 t), & L(x, y)>0 \\
\left(1-8 l_{x y}-4\left(l_{x y}+0.25\left(l_{x y}-2\right)^{2}\right) K^{+}\right)(1-0.5 t), & L(x, y) \leq 0,
\end{array},\right.
\end{aligned}
$$

where the source terms $q_{\alpha}$, porosity and viscosities are the same as Example 5.1. The entry pressure of capillary pressure is $p_{d}=1$. Dirichlet boundary condition on all of $\partial \Omega$ is imposed.

We test this example with two different case of permeability. Table 3 shows the $L^{2}, H^{1}$ errors of saturation, pressure and Darcy velocity when $T=1$ with permeability $\left(K^{+}=0.02, K^{-}=0.008\right)$ and Table 4 shows errors when $T=1$ with permeability $\left(K^{+}=0.1, K^{-}=0.001\right)$. The time step $\triangle t$ is chosen as $\triangle t=16 / n^{2}=\left(64 / \pi^{2}\right) h^{2}$.

The case of permeability $\left(K^{+}=0.02, K^{-}=0.008\right)$ shows second order in $L^{2}$-norm and first order in $H^{1}$-norm for the pressure variable, and first order in $L^{2}$-norm for the velocity variable. At least one and half order is observed in $L^{2}$-norm and around one half order in $H^{1}$-norm for the saturation.

The case of permeability $\left(K^{+}=0.1, K^{-}=0.001\right)$ shows similar convergence rates for the pressure and velocity variables. The average convergence rates for the saturation is 1.527 in $L^{2}$-norm and 0.455 in the $H^{1}$-norm. Here, an average convergence rate of $L^{2}$-norm is computed by

$$
\frac{\log \left(\left\|S_{w}-S_{w, h_{3}}\right\|_{L_{2}(\Omega)} /\left\|S_{w}-S_{w, h_{8}}\right\|_{L_{2}(\Omega)}\right)}{\log \left(h_{3} / h_{8}\right)},
$$

where $h_{k}=\pi / 2^{k+1}$. An average convergence rate of $H^{1}$-norm is computed similarly.

Example 5.4. In this example, we use the settings of five spot flooding problem in [3] (chapter 7.2.) for domain, parameters $\Phi, \lambda_{\alpha}$ and initial/boundary conditions, but we use different heterogeneities in the domain. 
Table 4: MFVM based on broken $Q_{1}$-nonconforming IFEM methods for Example 5.3 with permeability $\left(K^{+}=0.1, K^{-}=0.001\right)$

\begin{tabular}{|c|c|c|c|c|c|c|c|c|}
\hline Element & \multicolumn{2}{|c|}{$\left\|S_{w}-S_{w, h}\right\|_{L_{2}(\Omega)}$} & order & \multicolumn{2}{|c|}{$\left\|p-p_{h}\right\|_{L_{2}(\Omega)}$} & order & $\left\|\mathbf{u}-\mathbf{u}_{h}\right\|_{L_{2}(\Omega)}$ & order \\
\hline $8^{2}$ & \multicolumn{2}{|c|}{$2.636 \times 10^{-3}$} & & \multicolumn{2}{|c|}{$1.738 \times 10^{-2}$} & & \multicolumn{2}{|l|}{$1.069 \times 10^{-4}$} \\
\hline $16^{2}$ & \multicolumn{2}{|l|}{$1.282 \times 10^{-3}$} & 1.040 & \multicolumn{2}{|c|}{$3.972 \times 10^{-3}$} & 2.130 & $4.442 \times 10^{-}$ & 1.267 \\
\hline $32^{2}$ & \multicolumn{2}{|l|}{$3.132 \times 10^{-4}$} & 2.033 & \multicolumn{2}{|c|}{$9.503 \times 10^{-4}$} & 2.063 & $1.687 \times 10^{-}$ & 1.396 \\
\hline $64^{2}$ & \multicolumn{2}{|l|}{$2.260 \times 10^{-4}$} & 0.471 & \multicolumn{2}{|c|}{$2.272 \times 10^{-4}$} & 2.064 & $7.385 \times 10^{-}$ & 1.192 \\
\hline $128^{2}$ & \multicolumn{2}{|l|}{$3.328 \times 10^{-5}$} & 2.763 & \multicolumn{2}{|c|}{$5.819 \times 10^{-5}$} & 1.965 & $3.535 \times 10^{-}$ & 1.063 \\
\hline $256^{2}$ & \multicolumn{2}{|l|}{$1.326 \times 10^{-5}$} & 1.328 & \multicolumn{2}{|c|}{$1.434 \times 10^{-5}$} & 2.021 & $1.748 \times 10^{-6}$ & 1.016 \\
\hline & Element & \multicolumn{3}{|c|}{$\left\|\bar{S}_{w}-S_{w, h}\right\|_{1, h}$} & \multicolumn{3}{|c|}{$\left\|p-p_{h}\right\|_{1, h} \quad$ order } & \\
\hline & $8^{2}$ & \multicolumn{2}{|c|}{$5.553 \times 10^{-2}$} & & \multicolumn{3}{|c|}{$1.619 \times 10^{-1}$} & \\
\hline & $16^{2}$ & \multirow{2}{*}{\multicolumn{2}{|c|}{$4.702 \times 10^{-2}$}} & 0.240 & \multicolumn{3}{|c|}{$6.134 \times 10^{-2}$} & \\
\hline & $32^{2}$ & & & 0.817 & \multicolumn{3}{|c|}{$2.824 \times 10^{-2}$} & \\
\hline & $64^{2}$ & \multicolumn{2}{|c|}{$3.201 \times 10^{-2}$} & -0.262 & & \multirow{2}{*}{\multicolumn{2}{|c|}{$1.396 \times 10^{-2}$}} & \\
\hline & $128^{2}$ & \multicolumn{2}{|c|}{$1.618 \times 10^{-2}$} & 0.984 & & & 0.998 & \\
\hline & $256^{2}$ & \multicolumn{2}{|c|}{$1.147 \times 10^{-2}$} & 0.497 & \multicolumn{2}{|c|}{$3.501 \times 10^{-}$} & 0.998 & \\
\hline
\end{tabular}

We consider parameters

$$
\begin{cases}\Phi=0.2 & \\ \mu_{w}=0.001[\mathrm{~Pa} \cdot \mathrm{s}] & \mu_{n}=0.02[\mathrm{~Pa} \cdot \mathrm{s}] \\ \rho_{w}=1000\left[\mathrm{~kg} / \mathrm{m}^{3}\right] & \rho_{n}=1000\left[\mathrm{~kg} / \mathrm{m}^{3}\right] \\ K^{+}=10^{-10}\left[\mathrm{~m}^{2}\right], & K^{-}=10^{-14}\left[\mathrm{~m}^{2}\right] \\ L(x, y)=\left((x-85)^{2}+(y-185)^{2}\right)^{0.5}-50, & \end{cases}
$$

with initial condition,

$$
S_{w}(x, y, 0)= \begin{cases}0.8, & \text { if }(x, y) \in[30,140] \times[170.7,243.3] \\ 0, & \text { otherwise }\end{cases}
$$

Figure 7 shows $S_{w}$ at time $t=120[$ day $], t=240[$ day $]$ and $t=375[$ day $]$. The time step is chosen as $\triangle t=(1 / 240) h^{2}[$ day $]$.

\section{Conclusion}

We have introduced a new IMPES type of numerical method for two phase flows through heterogeneous porous media based on frameworks of IFEM and MFVM. Our scheme can be implemented to solve problems with heterogeneities of various shapes since we can use grids not necessarily aligning with the interface. The pressure variable is approximated by IFEM using Rannacher-Turek nonconforming element and Darcy velocity is obtained by the local recovery techniques of MFVM of Kwak [13, 29]. This is similar to, but different from the mixed hybrid method commonly used in porous media. We believe the MFVM technique in the context of [13] and IFEM technique are applied to the porous media problem for first time in this paper. Saturation equation is solved by the control volume method together with an upwinding scheme. The numerical results yield almost optimal orders of convergence in all variables for the zero capillary cases. For the nonzero capillary problem, convergence rates for the saturation deteriorate but are still optimal for other variables. 


\section{References}

[1] D. N. Arnold and F. Brezzi. Mixed and nonconforming finite element methods; implementation, postprocessing and error estimates. RAIROModélisation mathématique et analyse numérique, 19(1):7-32, 1985.

[2] D. N. Arnold, F. Brezzi, B. Cockburn, and L. D. Marini. Unified analysis of discontinuous galerkin methods for elliptic problems. SIAM journal on numerical analysis, 39(5):1749-1779, 2002.

[3] P. Bastian. Numerical computation of multiphase flows in porous media. Habilitationsschrift Univeristät Kiel, 1999.

[4] J. Bear. Dynamics of fluids in porous media. Elsevier, New York, 1972.

[5] F. Brezzi, J. Douglas Jr, M. Fortin, and L. D. Marini. Efficient rectangular mixed finite elements in two and three space variables. RAIROModélisation mathématique et analyse numérique, 21(4):581-604, 1987.

[6] F. Brezzi, J. Douglas Jr, and L. D. Marini. Two families of mixed finite elements for second order elliptic problems. Numerische Mathematik, 47(2):217-235, 1985 .

[7] F. Brezzi and M. Fortin. Mixed and hybrid finite element methods, volume 15. Springer-Verlag, New York, 1991.

[8] R. H. Brooks and A. T. Corey. Properties of porous media affecting fluid flow. In Journal of the Irrigation and Drainage Division, volume 92, pages 61-90, 1966 .

[9] K. S. Chang and D. Y. Kwak. Discontinuous bubble scheme for elliptic problems with jumps in the solution. Computer Methods in Applied Mechanics and Engineering, 200(5):494-508, 2011.

[10] G. Chavent and J. Jaffré. Mathematical models and finite elements for reservoir simulation: single phase, multiphase and multicomponent flows through porous media. Elsevier, 1986.

[11] G. Chavent and J. E. Roberts. A unified physical presentation of mixed, mixed-hybrid finite elements and standard finite difference approximations for the determination of velocities in waterflow problems. Advances in Water Resources, 14(6):329-348, 1991.

[12] S. H. Chou and D. Y. Kwak. Mixed covolume methods on rectangular grids for elliptic problems. SIAM Journal on Numerical Analysis, 37(3):758-771, 2000 .

[13] S. H. Chou, D. Y. Kwak, and K. Y. Kim. Mixed finite volume methods on nonstaggered quadrilateral grids for elliptic problems. Mathematics of computation, 72(242):525-539, 2003.

[14] S. H. Chou, D. Y. Kwak, and K. T. Wee. Optimal convergence analysis of an immersed interface finite element method. Advances in Computational Mathematics, 33(2):149-168, 2010. 
[15] M. Crouzeix and P. A. Raviart. Conforming and nonconforming finite element methods for solving the stationary stokes equations i. Revue française d'automatique, informatique, recherche opérationnelle. Mathématique, 7(3):33-75, 1973.

[16] J. Douglas and J. E. Roberts. Global estimates for mixed methods for second order elliptic equations. Mathematics of computation, 44(169):39 52,1985 .

[17] J. Douglas, Jr. Finite difference methods for two-phase incompressible flow in porous media. SIAM Journal on Numerical Analysis, 20(4):681-696, 1983.

[18] J. Douglas Jr, R. E. Ewing, and M. F. Wheeler. A time-discretization procedure for a mixed finite element approximation of miscible displacement in porous media. RAIRO-Analyse numérique, 17(3):249-265, 1983.

[19] L. J. Durlofsky. Accuracy of mixed and control volume finite element approximations to darcy velocity and related quantities. Water Resources Research, 30(4):965-973, 1994.

[20] L. Durlofsky J. A triangle based mixed finite elementfinite volume technique for modeling two phase flow through porous media. Journal of Computational Physics, 105(2):252-266, 1993.

[21] Y. Epshteyn and B. Riviere. Fully implicit discontinuous finite element methods for two-phase flow. Applied Numerical Mathematics, 57(4):383401, 2007.

[22] Y. Epshteyn and B. Rivière. Analysis of hp discontinuous galerkin methods for incompressible two-phase flow. Journal of Computational and Applied Mathematics, 225(2):487-509, 2009.

[23] R. E. Ewing. The Mathematics of Reservoir Simulation. SIAM, Philadelphia, 1983.

[24] R. E. Ewing, T. F. Russell, and M. F. Wheeler. Convergence analysis of an approximation of miscible displacement in porous media by mixed finite elements and a modified method of characteristics. Computer Methods in Applied Mechanics and Engineering, 47(1):73-92, 1984.

[25] P. A. Forsyth. A control volume finite element approach to napl groundwater contamination. SIAM Journal on Scientific and Statistical Computing, 12(5):1029-1057, 1991.

[26] R. Helmig. Multiphase flow and transport processes in the subsurface: a contribution to the modeling of hydrosystems. Springer-Verlag, 1997.

[27] R. Huber and R. Helmig. Multiphase flow in heterogeneous porous media: A classical finite element method versus an implicit pressure-explicit saturation-based mixed finite element-finite volume approach. International journal for numerical methods in fluids, 29(8):899-920, 1999. 
[28] J. Kou and S. Sun. Upwind discontinuous galerkin methods with mass conservation of both phases for incompressible two-phase flow in porous media. Numerical Methods for Partial Differential Equations, 30(5):16741699, 2014.

[29] D. Y. Kwak, K. T. Wee, and K. S. Chang. An analysis of a broken $p_{1^{-}}$ nonconforming finite element method for interface problems. SIAM Journal on Numerical Analysis, 48(6):2117-2134, 2010.

[30] Z. Li, T. Lin, Y. Lin, and R. C. Rogers. An immersed finite element space and its approximation capability. Numerical Methods for Partial Differential Equations, 20(3):338-367, 2004.

[31] Z. Li, T. Lin, and X. Wu. New cartesian grid methods for interface problems using the finite element formulation. Numerische Mathematik, 96(1):61-98, 2003.

[32] A. Michel. A finite volume scheme for two-phase immiscible flow in porous media. SIAM Journal on Numerical Analysis, 41(4):1301-1317, 2003.

[33] C. T. Miller, G. Christakos, P. T. Imhoff, J. F. McBride, J. A. Pedit, and J. A. Trangenstein. Multiphase flow and transport modeling in heterogeneous porous media: challenges and approaches. Advances in Water Resources, 21(2):77-120, 1998.

[34] D. W. Peaceman. Fundamentals of numericl reservoir simulation. Elsevier North-Holland, Inc., New York, 1977.

[35] R. Rannacher and S. Turek. Simple nonconforming quadrilateral stokes element. Numerical Methods for Partial Differential Equations, 8(2):97$111,1992$.

[36] P. A. Raviart and J. M. Thomas. A mixed finite element method for 2-nd order elliptic problems. Mathematical aspects of finite element methods, pages $292-315,1977$.

[37] L. Ronghua. Generalized difference methods for a nonlinear dirichlet problem. SIAM journal on numerical analysis, 24(1):77-88, 1987.

[38] T. F. Russell and M. F. Wheeler. Finite element and finite difference methods for continuous flows in porous media. The mathematics of reservoir simulation, 1:35-106, 1983.

[39] J. W. Sheldon and W. T. Cardwell Jr. One-dimensional, incompressible, noncapillary, two-phase fluid flow in a porous medium. Petroleum Transactions, AIME, 216:290-296, 1959.

[40] H. L. Stone and A. O. Garder Jr. Analysis of gas-cap or dissolved-gas drive reservoirs. Society of Petroleum Engineers Journal, 1(02):92-104, 1961.

[41] M. F. Wheeler. An elliptic collocation-finite element method with interior penalties. SIAM Journal on Numerical Analysis, 15(1):152-161, 1978. 